\title{
Burnout, satisfacción y calidad de vida laboral en funcionarios de la salud pública chilenos
}

\author{
Burnout syndrome, satisfaction, and quality of work life in Chilean public health workers \\ Jorge Alejandro Salgado-Roa' ${ }^{1}$ orcid.org/0000-0003-2470-4988 \\ Francisco José Lería-Dulčić1* orcid.org/0000-0001-6326-2567
}

1 Departamento de Psicología e Instituto de Investigación en Ciencias Sociales y Educación, Universidad de Atacama. Copiapó, Chile

\section{Resumen}

Introducción: Si bien se ha constatado la influencia negativa del síndrome de quemarse en el trabajo o Síndrome de Burnout (SB) en el desempeño laboral, aun los datos son elusivos respecto de su relación específica con la Satisfacción Laboral (SL) y Calidad de Vida Profesional (CVP) en el contexto de las profesiones asociadas a la salud pública. Objetivo: Analizar la asociación entre SB, SL y CVP, y algunas variables sociolaborales de 169 profesionales de la salud pública chilena. Materiales y métodos: Estrategia asociativa comparativa-transversal, aplicando el Inventario de Burnout de Maslach (MBI), la Escala de Satisfacción Laboral (S20/23), y el Cuestionario de Vida Profesional (CVP-35). Resultados: Un $10 \%$ de los trabajadores percibió altos niveles de SB, y una SL y CVP satisfactorias; un 88,8\% medio/moderado. La edad cronológica, Motivación Intrínseca, Apoyo Directivo, estado civil y años de servicio explicaron el 33,5\% de la variabilidad del Cansancio Emocional; y se plantean buenos predictores del SB. Conclusiones: Los datos reflejan una prevalencia media/moderada de SB, que puede ser entendida a la luz de la influencia insidiosa del síndrome. La edad cronológica y el estado civil se plantean como factores protectores. Se discuten los resultados en el contexto de la literatura existente.

Palabras clave: Agotamiento psicológico; satisfacción en el trabajo; calidad de vida; estrés laboral; trabajadores de la salud. (Fuente: DeCS, Bireme).

\begin{abstract}
Introduction: The negative influence of the burnout syndrome (BS) on work performance has been demonstrated. However, data are still elusive regarding its specific relationship with Job Satisfaction (JS) and Professional Quality of Life (ProQOL) in professions associated with public health. Objective: To analyze the association between BS, JS and ProQOL, and some socio-labor variables of 169 Chilean public health professionals. Materials and methods: A comparative crosssectional associative study that applied the Maslach Burnout Inventory (MBI), the Labor Satisfaction Scale (S20/23), and the Professional Life Questionnaire (PLQ-35). Results: 10\% of participants perceived high BS levels and satisfactory HS and ProQOL, while $88.8 \%$ of health professionals acknowledged medium/moderate levels. $33.5 \%$ of the variability in Emotional Fatigue could be explained by chronological age, Intrinsic Motivation, Management Support, marital status and years of service. Good BS predictors are suggested. Conclusions: The data reflect a medium/moderate prevalence of BS, which can be understood in light of the insidious influence of this syndrome. Chronological age and marital status are suggested as protective factors. The results are discussed in the context of current literature.
\end{abstract}

Key words: Burnout; job satisfaction; quality of life; occupational stress; healthcare workers. (Source: DeCS, Bireme).

\footnotetext{
*Autor de correspondencia

Francisco José Lería Dulčić

e-mail: francisco.leria@uda.cl
} 


\section{Introducción}

Los estudios acerca de los riesgos psicosociales en el trabajo han sido motivo de una larga tradición investigativa, revelando el impacto que poseen los trastornos ansiosos en el desempeño laboral del trabajador(1). Uno de los más estudiados, el síndrome de Burnout (SB), definido como un estado emocional crónico y desadaptativo afrontamiento al estrés, que conduce a extenuación y distanciamiento emocional en el trabajo(2). Este ha sido explorado en un amplio espectro de profesiones asistenciales ${ }^{(3,4)}$, incluyendo aquellas asociadas al ámbito de la salud(5,6), con una prevalencia variable para los distintos países, así a nivel internacional es de aproximadamente un $21 \%(4) ;$ y en los países latinoamericanos, oscila desde un $12,5 \%$ hasta incluso un $43,82 \%$ (7). Estudios en el área han sugerido una serie de factores protectores y de riesgo específicos que hacen más propensas a estas profesiones para su desarrollo, existiendo evidencia que indica una asociación con la calidad de vida profesional (CVP) y la satisfacción laboral (SL) ${ }^{(8,9)}$.

Desde la conceptualización inicial establecida por el modelo de Maslach, el SB se ha entendido como una dificultad manifiesta para el afrontamiento al estrés crónico a consecuencia de las características del trabajo. Comprende la presencia de sintomatología como fatiga física y emocional, decaimiento, irritabilidad y depresión, se manifiesta en personas sin psicopatología premórbida y provoca un desempeño laboral deficiente debido a actitudes y disposiciones negativas al trabajo. Este modelo comprende tres subdimensiones: Agotamiento Emocional, Despersonalización o Cinismo, y Disminución del Desempeño Personal o Falta de Realización Personal(10). El Agotamiento Emocional representa el aspecto individual del SB, y hace referencia a la percepción que tiene el trabajador de quedarse sin recursos ni energías, que se exterioriza con síntomas físicos y emocionales como irritabilidad, ansiedad y cansancio.

La Despersonalización o Cinismo alude al contexto interpersonal del SB y da cuenta del desarrollo de actitudes negativas e insensibilidad hacia las personas con las cuales el trabajador se relaciona, derivando en conflictos y aislamiento. La presencia de este factor se constituye en un impedimento para quienes ejercen profesiones de ayuda y servicio a personas, dada la necesidad de conexión, contacto y empatía con los usuarios y sus demandas(11). Finalmente, la Falta de Realización Personal, indica el aspecto autoevaluativo del SB y destaca la sensación de los trabajadores de que no están obteniendo logros en su trabajo. Por su parte, la CVP es una medida de los factores emocionales, que es definida como un estado emocional positivo resultante de la percepción subjetiva y el conjunto de sentimientos favorables y desfavorables respecto del trabajo(12). La SL es una medida fundamentalmente cognitiva que hace referencia a un juicio y/o valoración global sobre la experiencia vital que una persona tiene de sí misma en el trabajo(13).

Los profesionales de la salud chilenos que ejercen en atención primaria en los denominados Centros de Salud Familiar Municipal (CESFAM), están regularmente sometidos a condiciones altamente demandantes de desempeño, particularmente por el exceso de trabajo y número de usuarios atendidos diariamente; esto se explica a razón de la fatiga propia por las prolongadas horas de trabajo y permanencia en las actividades profesionales, que muchas veces exceden lo legalmente establecido, encontrándose así el profesional de la salud con muchas de las variables propicias para la aparición y desarrollo del SB(14,15). Este tipo de centros de salud llegan a más de 250 en el país, existiendo entre ellos notables diferencias por los lugares geográficos en los que se encuentran por la naturaleza económica, social, cultural o racial de las personas que son atendidas, lo que intensifica la demanda asistencial y su complejidad. Por ejemplo, el CESFAM más grande del país atiende aproximadamente 60000 usuarios, donde el $30 \%$ de sus funcionarios han tenido alguna licencia médica de tipo psiquiátrica(16).

En Chile existe evidencia vinculada a la prevalencia del SB en los trabajadores de este tipo de establecimientos de salud pública, con resultados que alcanzan un $27,8 \%{ }^{(17)}$ y hasta un alarmante $81 \%$ de trastornos ansiosos(18). Además, datos cualitativos destacan la relación entre la percepción de estos trabajadores con las condiciones adversas de desempeño, la autopercepción de estrés, un clima laboral adverso y una baja SL(19); denotando el complejo y multivariado rol de las variables psicosociales pertenecientes a los contextos laborales y políticas públicas que los sostienen.

Considerando que las dimensiones cognitivas y emocionales del trabajador juegan un papel esencial 
en la generación del SB(20), por ejemplo, en la influencia negativa sobre la satisfacción y calidad de vida(21); y/o por el contrario, el rol preventivo del capital psicológico necesario para su afrontamiento efectivo(22); aún los resultados se muestran un tanto elusivos.

Particularmente, los trabajadores de la salud pública latinoamericanos se han enfrentado a importantes cambios en los últimos años, en términos del aumento de la cobertura y población que es atendida en estos centros asistenciales, con el consecuente impacto psicológico a nivel individual.

No obstante, la escasez de evidencia empírica actualizada hace de su indagación un aspecto relevante a considerar en la exploración de las características que adquiere el SB en estos colectivos laborales y contextos socioeconómicos que les son propios. Datos obtenibles en espacios urbanos de mediano tamaño con sus características locales específicas (sociodemográficas, culturales y/o circunstanciales), permitiría ahondar en lo planteado por algunos estudios multicéntricos, respecto de las diferencias propias y elementos comunes del SB en función de la localidad geográfica(23). Además, explorar las características de las variables en estudio en esta población permitiría identificar factores que pudiesen intensificar o amortizar la mantención del SB en el tiempo; y así incluirlas en las decisiones de las respectivas directivas frente al afrontamiento a esta problemática.

El objetivo del estudio consiste en analizar la asociación entre SB y dos medidas de autopercepción, una emocional asociada al conjunto de sentimientos favorables y desfavorables respecto del trabajo (CVP), y otra cognitiva, asociada a la valoración global sobre la experiencia vital en el trabajo (SL).

\section{Materiales y métodos}

\section{Diseño}

Empírico con una estrategia asociativa de tipo comparativa transversal y diseño de grupos naturales. No se manipulan variables y se analizan sus relaciones indagando las diferencias entre dos o más grupos de individuos.

\section{Participantes}

Muestra no probabilística por conveniencia de 169 funcionarios provenientes de tres CESFAM de la ciudad de Copiapó, ciudad ubicada en el norte de Chile con aproximadamente 200000 habitantes. En la muestra se incluyó auxiliares, técnicos y profesionales universitarios, 99 mujeres $(58,6 \%)$ y 70 hombres $(41,4 \%)$, con edades entre 20 y 60 años $(\mathrm{M}=36,8, \mathrm{DE}=9,84) ; \mathrm{y}$ un promedio de años de servicio de 8,08 (DE=7,91).

\section{Instrumentos}

Para identificar el nivel de SB se empleó la versión catalana del Inventario de Burnout de Maslach (MBI, Maslach Burnout Inventory)(24). Este instrumento corresponde a una escala Likert que indica la frecuencia con la que se experimenta el SB durante el último año, según los siguientes rangos: 1 (Nunca); 2 (Algunas veces al año); 3 (Algunas veces al mes); 4 (Algunas veces a la semana); y, 5 (Diariamente). EL MBI consta de veintidós ítems distribuidos en tres escalas: Cansancio emocional, (CE), que consta a su vez de nueve ítems que evalúan la vivencia de estar exhausto a nivel emocional debido a las demandas del trabajo; despersonalización, (DP), constituida por cinco ítems, que mide el grado de frialdad y distanciamiento relacional en el trabajo; y realización personal, (RP), con ocho ítems que evalúan los sentimientos de eficacia, competencia y realización de los objetivos personales en el trabajo.

El MBI presenta alta consistencia interna y fiabilidad en sus tres dimensiones $y$ sus propiedades psicométricas arrojan valores de consistencia interna aceptables de Agotamiento Emocional $(\alpha=0,64) ; \quad$ Despersonalización $\quad(\alpha=0,75) ; \quad y$ Realización Personal $(\alpha=0,83)^{(25)}$, así como una alta consistencia para la puntuación global $(\alpha=0,81)$; siendo un instrumento válido y confiable, debido a su capacidad discriminatoria y estructura factorial. Los puntos de corte se establecen con base a la media teórica, como lo han realizado varios de los estudios citados.

Para establecer el nivel de CVP se aplicó el Cuestionario de Calidad de Vida Profesional (CVP35) de Cabezas, versión en castellano del PQL-35 de

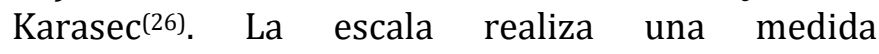
multidimensional de la CVP, a través cuatro subescalas (apoyo directivo; cargas de trabajo; motivación intrínseca y calidad de vida), que hacen referencia a la percepción que tiene el trabajador de las condiciones de su puesto de trabajo. Se conforma con 35 ítems que ofrecen respuestas de opinión en una escala de 1 a 10 puntos: Deficiente (1 a 2 
puntos); Regular (3 a 5 puntos), Buena (6 a 8 puntos), y Excelente (9 a 10 puntos). El cuestionario se conforma por tres dimensiones: apoyo directivo (13 ítems), cargas de trabajo (11 ítems) y motivación intrínseca (10 ítems). El ítem 35 se valora de manera independiente e identifica la percepción de la calidad de vida en el trabajo.

Por último, para determinar el nivel de SL, se aplicó la Escala de Satisfacción Laboral (S20/23) de Meliá y Peiró(27), elaboró a partir del cuestionario de Satisfacción Laboral S4/82, consta de 23 ítems y valora 5 factores: supervisión; ambiente físico del trabajo; prestaciones recibidas; satisfacción intrínseca del trabajo y; satisfacción con la participación. Posee las alternativas de respuesta: 1 (Muy insatisfecho), 2 (Bastante insatisfecho), 3 (Algo insatisfecho), 4 (Indiferente), 5 (Algo satisfecho), 6 (Bastante satisfecho), y, 7 (Muy satisfecho), a pesar de constar de 59 ítems menos, el cuestionario presenta un $\alpha$ de 0,92 y correlaciona 0,92 con el S4/82(28). Finalmente, se realizaron algunas preguntas orientadas a recolectar información sociodemográfica de los y las participantes, respecto del estado civil, edad, nivel de ingreso familiar y nivel educacional.

\section{Procedimientos}

Para describir las diferentes dimensiones del SB, CVP y SL, se calcularon sus promedios y desviaciones estándar, así como las correlaciones lineales de Pearson $(r)$ entre las dimensiones que componen el MBI, CVP-35 y el S20/23. Luego, se analizaron las diferencias de los puntajes totales de los instrumentos aplicados y sus dimensiones en función de las variables sociodemográficas (sexo, edad, estado civil, ingreso familiar y nivel de estudios). Para el sexo, se utilizó la prueba de $t$ de Student para muestras independientes. En el caso de no existir homogeneidad, se aplicó el test de Welch. Para el resto de las variables, se realizó un análisis de varianza unifactorial ANOVA. Las comparaciones post-hoc se realizaron aplicando la prueba de Scheffé y para determinar el tamaño del efecto se calculó la d de Cohen (d) y el Eta Cuadrado Parcial $\left(\eta_{p}{ }^{2}\right)^{(29)}$.

Finalmente, se ingresó a un modelo de regresión lineal múltiple, con el método por pasos (stepwise), las puntuaciones de las dimensiones del MBI como variables dependientes y las variables edad, años de servicio y estado civil como variables independientes. Además, se incorporan las dimensiones del CVP-35 y el S20/23 al análisis de regresión. El procesamiento estadístico se realizó con el software SPSS 23.0.

\section{Consideraciones éticas}

Se solicitó la autorización de los directores de los CESFAM para proceder a recolectar los consentimientos informados firmados por los participantes del estudio. Posteriormente, se dio a conocer los objetivos del estudio y el carácter confidencial de la información proporcionada, así como de los riesgos mínimos existentes. La participación fue voluntaria y no hubo incentivos. El estudio aprobado por el Comité de Ética Científica de la Universidad de Atacama (Chile), resguardando el cumplimiento de los aspectos éticos propuestos por la Asociación Americana de Psicología (APA).

\section{Resultados}

Las características sociodemográficas de la muestra, niveles de Burnout, Calidad de Vida Profesional y Satisfacción Laboral se describen en la Tabla 1.

Tabla 1. Descriptivos de las características sociodemográficas de la muestra

\begin{tabular}{lr}
\hline \multicolumn{1}{c}{ Variable } & N (\%) \\
\hline Estado civil & $79(46,7)$ \\
Soltero & $64(37,9)$ \\
Casado & $4(2,4)$ \\
Viudo & $22(13)$ \\
Separado/divorciado & \\
Edad & $62(36,7)$ \\
20 a 30 años (1) & $49(29)$ \\
31 a 40 años (2) & $33(19,5)$ \\
41 a 50 (3) & $25(14,8)$ \\
51 a 60 (4) & \\
Nivel de ingreso familiar & $2(1,2)$ \\
Menos de \$250.000 & $59(34,9)$ \\
Entre \$251.000 y \$500.000 & $58(34,3)$ \\
Entre \$501.000 y un millón & $50(29,6)$ \\
Más de un millón & \\
Nivel educacional & $25(14,8)$ \\
Ed. media (sin título, auxiliares) & $76(45)$ \\
Ed. media técnica/profesional (título, técnicos) & $68(40,2)$ \\
Educación universitaria &
\end{tabular}

Los datos indican que 18 personas se ubican en un rango de Alto Burnout (10,7\%) y 150 en el nivel de Burnout Medio/Moderado (88,8\%), observándose las puntuaciones más elevadas en la dimensión de realización personal y cansancio emocional. Respecto a la CVP existe un alto nivel que supera la media teórica y los valores más altos se ubican en las dimensiones de Apoyo Directivo y Carga de Trabajo. 
Así también, la SL presenta una media teórica superior en las siguientes dimensiones; satisfacción con las prestaciones recibidas, satisfacción con el ambiente físico y satisfacción con la supervisión (Tabla 2).

Tabla 2. Descriptivos de las escalas totales y dimensiones del MBI, CVP35 y S20/23

\begin{tabular}{lll}
\hline \multicolumn{1}{c}{ Total/Dimensión } & \multicolumn{1}{c}{ Media } & \multicolumn{1}{c}{$\boldsymbol{D E}$} \\
\hline MBI & 64,12 & 8,18 \\
Cansancio emocional & 24,53 & 6,16 \\
Despersonalización & 11,47 & 3,62 \\
Realización personal & 28,31 & 5,24 \\
CVP35 & 219,56 & 36,79 \\
Apoyo directivo & 79,16 & 14,24 \\
Carga de trabajo & 71,21 & 12,82 \\
Motivación intrínseca & 49,52 & 13,71 \\
Calidad de vida & 19,67 & 5,17 \\
S20/23 & 111,91 & 27,8 \\
Satisfacción con la supervisión & 23,57 & 7,35 \\
Satisfacción con el ambiente físico & 24,38 & 7,44 \\
Satisfacción con las prestaciones Recibidas & 25,47 & 5,82 \\
Satisfacción intrínseca & 19,54 & 6,13 \\
Satisfacción con la participación & 18,93 & 5,81 \\
\hline
\end{tabular}

Existe correlación significativa positiva entre el Cuestionario de Satisfacción Laboral y el de Calidad de Vida Profesional $r(169)=0,203, p=0,008$. No se observa correlación entre el MBI y las otras dos escalas. Respecto de las correlaciones entre las dimensiones que componen el MBI y el CVP-35, (Tabla 3) las más fuertes e indirectas se presentan entre cansancio emocional con motivación intrínseca y calidad de vida. Por otra parte, se observa la presencia de correlaciones positivas entre realización personal con carga de trabajo y motivación intrínseca.

Las correlaciones entre las Dimensiones del MBI y el S20/23, las más altas e indirectas, se observan entre cansancio emocional y satisfacción con la supervisión $[r(168)=-0,343, p=0,001]$; satisfacción con prestaciones recibidas $[r(169)=-0,28, p=0,001]$; satisfacción intrínseca $[r(169)=-0,24, p=0,001]$; y satisfacción con la participación $[r(169)=-0,28$, $p=0,001]$. Por otra parte, la dimensión despersonalización correlaciona de manera positiva con satisfacción con el ambiente físico $[r(168)=0,26$, $p=0,001]$, y con satisfacción con prestaciones recibidas $[r(168)=0,22, p=0,001]$.

Respecto de las correlaciones entre las Dimensiones del CVP-35 y S20/23, se observa que las más altas se presentan entre Motivación Intrínseca y Satisfacción con la Supervisión $[r(169)=0,46, \quad p=0,001], \quad y$ Satisfacción con las Prestaciones Recibidas $[r(169)=0,4, p=0,001]$. Así también, entre Motivación Intrínseca y Satisfacción con la Participación $[r(169)=0,54, p=0,001]$. Por otra parte, se observa una correlación indirecta entre Apoyo Directivo y Satisfacción con el Ambiente Físico $[r(169)=-0,3$, $p=0,001]$.

Diferencias en el Burnout, calidad de vida profesional y satisfacción laboral en función de las variables sociodemográficas

En relación al $S B$, CVP y SL en función del sexo, no se presentan diferencias en las puntuaciones obtenidas por ambos grupos. Respecto a las diferencias entre las Dimensiones del MBI, CVP y SL en función del sexo, se observan medias desiguales, siendo mayor en los hombres la despersonalización $[t(167)=2,67$, $p=0,008, d=0,42,95 \%$ IC $(0,38,2,59)] y$ en las mujeres la realización personal $[t(167)=-2,4, p=0,01$, $d=0,38,95 \%$ IC $(-3,55,-0,35)]$ y la motivación intrínseca $[t(167)=-2,47, p=0,01, d=0,39,95 \%$ IC $(-$ $9,39,-1,06)]$. Cabe destacar que el tamaño del efecto en las tres diferencias señaladas fue de moderado a pequeño ( $d$ de Cohen entre 0,38 y 0,42 ), lo que significa que la variable Sexo genera diferencias significativas en las dimensiones del MBI, CVP35 y S20/23.

Tabla 3. Correlaciones entre las dimensiones del MBI y el CVP-35

\begin{tabular}{|c|c|c|c|c|c|c|c|}
\hline $\begin{array}{c}\text { Dimensión } \\
\text { MBI/CVP-35 }\end{array}$ & CE & DP & $\mathbf{R P}$ & AD & CT & MI & CV \\
\hline Cansancio Emocional (CE) & -- & $0,22^{* *}$ & $-0,09$ & $-0,06$ & $-0,07$ & $-0,43^{* *}$ & $-0,36^{* *}$ \\
\hline Despersonalización (DP) & & -- & $-0,18^{*}$ & $-0,01$ & 0,04 & $-0,14$ & 0,07 \\
\hline Realización Personal (RP) & & & - & 0.09 & $0,2^{* *}$ & $0,28^{* *}$ & 0,05 \\
\hline Apoyo Directivo (AD) & & & & -- & $0,72^{* *}$ & $0,41^{* *}$ & $0,2^{* *}$ \\
\hline Carga de Trabajo (CT) & & & & & -- & $0,56^{* *}$ & $0,35^{* *}$ \\
\hline Motivación Intrínseca (MI) & & & & & & -- & $0,47^{* *}$ \\
\hline Calidad de Vida (CV) & & & & & & & -- \\
\hline
\end{tabular}


Respecto de las diferencias en las escalas $y$ dimensiones del MBI, CVP-35 y S20/23 en función del rango etario, existen desigualdades en las puntuaciones del cuestionario de CVP-35 $\left[F(3,16)=3,24, p<0,05, \eta_{p}{ }^{2}=0,05,95 \%\right.$ ICs $(200,01$, 218,28), (211,79; 229,88), (216,03; 242,75) y $(212,47 ; 247,29)$, respectivamente], en las dimensiones de Apoyo directivo $[F(3,165)=2,96$, $p<0,05, \eta_{p}{ }^{2}=0,051,95 \%$ ICs $(71,54 ; 79,04)$, (76,49; respectivamente], y Carga de trabajo $\left[F(3,165)=3,577, p<0,05, \eta_{p}{ }^{2}=0,061,95 \%\right.$ ICs $(65,41$; $71,27),(66,55 ; 73,08),(71,33 ; 80,97)$ y $(68,17$; 80,87)].

Se aprecia un efecto moderado a bajo con un Eta parcial cuadrado entre 0,061 y 0,05 que significa que la variable rango etario genera diferencias medianamente significativas en el CVP-35 y las dimensiones mencionadas respectivamente. Al aplicar la prueba post hoc de Scheffe se observan diferencias entre el Rango 1 y el Rango $3(p<0,05)$ con respecto a la Carga de trabajo, lo cual significa que los participantes con edades entre 41 y 50 años, perciben una mayor carga laboral que aquellos entre los 20 y 30 años.

Con relación a las diferencias en las escalas y dimensiones del MBI, CVP-35 y S20/23 en función del Estado Civil, se presentan desigualdades en la escala total del MBI $[F(3,164)=3,68, p<0,01$, $\eta_{p}{ }^{2}=0,06,95 \%$ ICs $(63,03 ; 66,82),(62,85 ; 67,09)$, $(54,32 ; 78,18)$ y $(54,11 ; 62,80)$, respectivamente], y en la dimensión de Cansancio emocional $[F(3$, $165)=5,82, \quad p<0,001, \quad \eta_{\mathrm{p}}^{2}=0,099,95 \%$ ICs $(23,66$; $26,49),(23,92 ; 26,71),(20,95 ; 34,55)$ y $(17,30$; $22,15)]$. Se aprecia un tamaño del efecto moderado respecto de la escala total y la dimensión señalada. Así también, se presentan diferencias en la dimensión Satisfacción con las Prestaciones recibidas del S20/23 $[F(3,165)=3,1, \quad p<0,05$, $\eta_{p}{ }^{2}=0,05,95 \%$ ICs $(23,01,25,85),(25,45 ; 27,83)$, $(4,88 ; 36,12)$ y $(24,45 ; 28,91)]$. Al aplicar la prueba post hoc de Scheffe se observan diferencias entre el grupo de soltero y separado/divorciado $(p<0,05)$ y el grupo de casado con Separado/divorciado $(p<0,05)$ con respecto al puntaje total del MBI y de la Dimensión de Cansancio Emocional.

Respecto a las diferencias en las escalas $y$ dimensiones del MBI, CVP-35 y S20/23 en función del Nivel Educacional, se presentan diferencias en la dimensión de Cansancio emocional $[F(2,166)=4,11$, $p<0,05, \eta_{p}{ }^{2}=0,04,95 \%$ ICs $(19,37 ; 25,83),(24,70$; $27,22)$ y $(22,22 ; 25,08)$, respectivamente]. Así también, en la dimensión Calidad de vida del CVP-35 $\left[F(2,16)=6,29, p<0,01, \quad \eta_{p}^{2}=0,06,95 \%\right.$ ICs $(16,86$; $21,06),(17,20 ; 19,64)$ y $(20,21 ; 22,44)]$. Se aprecia un tamaño del efecto moderado respecto de la Calidad de vida del CVP-35 $\left(\eta_{p}^{2}=0,069\right)$. Al aplicar la prueba post hoc de Scheffe se observan diferencias entre el grupo de participantes con título universitario y el grupo con nivel técnico $(p<0,05)$ en la dimensión Calidad de vida, donde los primeros perciben una mejor calidad de vida que los participantes con nivel técnico.

En relación a las diferencias de medias en función de la variable ingreso familiar, no se presentan desigualdades en los puntajes totales ni en las dimensiones que las componen.

Las dimensiones del MBI fueron ingresadas a un modelo de regresión lineal como variables dependientes, y se consideró la edad, años de servicio y estado civil como variables independientes. Además, se incorporó por separado cada una de las dimensiones que componen en CVP35 y el S20/23. Respecto a cansancio emocional (Tabla 4) existió la formación de cinco modelos predictores. El primer modelo observado tuvo 18\% de la varianza explicada por motivación intrínseca. El último modelo, con una varianza explicada de $36 \%$, tiene un mayor peso de los factores motivación intrínseca, apoyo directivo, calidad de vida, y de las variables estado civil y edad, que representan, respectivamente, una percepción negativa de calidad de vida laboral, especialmente en personas solteras, y a su vez baja motivación personal. Con relación a realización personal, se generaron dos modelos, siendo el más significativo el que ingreso el factor motivación intrínseca y años de servicio, que explicaron el $11,3 \%$ de la varianza del puntaje de la dimensión, lo que significa que el aumento de los dos predictores eleva la dimensión realización personal.

Al incorporar las dimensiones del S20/23 al modelo de regresión (Tabla 5), los valores más significativos se presentan respecto a cansancio emocional, y los predictores ingresados fueron satisfacción con la supervisión, estado civil y años de servicio, explicando el 18,8\% de la varianza del puntaje de la dimensión. Por lo tanto, los trabajadores que perciben negativamente la supervisión en el trabajo, por un tiempo prolongado y pertenecen al rango de 
Tabla 4. Análisis de regresión por el Método Stepwise, entre los Factores del CVP-35, datos sociodemográficos y dimensiones del MBI

\begin{tabular}{|c|c|c|c|c|c|c|c|c|}
\hline \multirow{2}{*}{$\begin{array}{c}\text { Dimensiones } \\
\text { MBI }\end{array}$} & \multirow{2}{*}{ Modelo } & \multirow{2}{*}{$\begin{array}{c}\text { Predictores } \\
\text { Sd/ Factores } \\
\text { CVP-35 }\end{array}$} & \multicolumn{2}{|c|}{ Coef. no estandarizados } & \multirow{2}{*}{$\begin{array}{c}\text { Coef. } \\
\text { Estandarizados } \\
\beta\end{array}$} & \multirow{2}{*}{$R^{2}$} & \multirow{2}{*}{$F($ gl, n-gl) } & \multirow{2}{*}{$P$} \\
\hline & & & B & EE & & & & \\
\hline Cansancio & 1 & (Const.) & 34,17 & 1,61 & & & & \\
\hline \multirow[t]{19}{*}{ Emocional } & & MI & $-0,19$ & 0,03 & $-0,43$ & 0,18 & $38,6(1,16)$ & 0,001 \\
\hline & 2 & (Const.) & 26,87 & 2,39 & & & & \\
\hline & & MI & $-0,25$ & 0,03 & $-0,55$ & 0,25 & $28,91(2,16)$ & 0,001 \\
\hline & & $\mathrm{AD}$ & 0,12 & 0,03 & 0,29 & & & \\
\hline & 3 & (Const.) & 28,78 & 2,4 & & & & \\
\hline & & MI & $-0,24$ & 0,03 & $-0,54$ & 0,3 & $24(3,16)$ & 0,001 \\
\hline & & $\mathrm{AD}$ & 0,13 & 0,03 & 0,29 & & & \\
\hline & & EC & $-1,32$ & 0,4 & $-0,21$ & & & \\
\hline & 4 & (Const.) & 31,74 & 2,52 & & & & \\
\hline & & MI & $-0,19$ & 0,03 & $-0,43$ & 0,34 & $21,42(4,16)$ & 0,001 \\
\hline & & $\mathrm{AD}$ & 0,13 & 0,03 & 0,3 & & & \\
\hline & & $\mathrm{EC}$ & $-1,41$ & 0,39 & $-0,22$ & & & \\
\hline & & $\mathrm{CV}$ & $-0,26$ & 0,08 & $-0,22$ & & & \\
\hline & 5 & (Const.) & 29,45 & 2,66 & & & & \\
\hline & & MI & $-0,2$ & 0,03 & $-0,45$ & 0,36 & $18,78(5,16)$ & 0,001 \\
\hline & & $\mathrm{AD}$ & 0,12 & 0,03 & 0,27 & & & \\
\hline & & EC & $-1,83$ & 0,42 & $-0,29$ & & & \\
\hline & & $\mathrm{CV}$ & $-0,25$ & 0,08 & $-0,21$ & & & \\
\hline & & Edad & 0,1 & 0,04 & 0,16 & & & \\
\hline \multirow{5}{*}{$\begin{array}{l}\text { Realización } \\
\text { Personal }\end{array}$} & 1 & (Const.) & 22,98 & 1,45 & & & & \\
\hline & & MI & 0,1 & 0,02 & 0,28 & 0,08 & $14,38(1,16)$ & 0,001 \\
\hline & 2 & (Const.) & 22,01 & 1,48 & & & & \\
\hline & & MI & 0,1 & 0,02 & 0,28 & 0,11 & $10,54(2,16)$ & 0,001 \\
\hline & & AS & 0,12 & 0,04 & 0,18 & & & \\
\hline
\end{tabular}

$\mathrm{MI}$ = Motivación Intrínseca; AD = Apoyo Directivo; EC = Estado Civil; CV = Calidad de Vida; AS = Años de Servicio

Tabla 5. Análisis de Regresión por el Método Stepwise, entre los Factores del S20/23, Datos Sociodemográficos y Dimensiones del MBI

\begin{tabular}{|c|c|c|c|c|c|c|c|c|}
\hline \multirow{2}{*}{$\begin{array}{c}\text { Dimensiones } \\
\text { MBI }\end{array}$} & \multirow{2}{*}{ Modelo } & \multirow{2}{*}{$\begin{array}{l}\text { Predictores } \\
\text { Sd/ Factores } \\
\text { S20/23 }\end{array}$} & \multicolumn{2}{|c|}{ Coef. no estandarizados } & \multirow{2}{*}{$\begin{array}{c}\text { Coef. } \\
\text { Estandarizados } \\
\beta \\
\end{array}$} & \multirow{2}{*}{$R^{2}$} & \multirow{2}{*}{$F(g l, n-g l)$} & \multirow{2}{*}{$P$} \\
\hline & & & B & EE & & & & \\
\hline Cansancio & 1 & (Const.) & 31,3 & 1,5 & & & & \\
\hline \multirow[t]{8}{*}{ Emocional } & & SS & $-0,28$ & 0,06 & $-0,34$ & 0,11 & $22,245(1,16)$ & 0,001 \\
\hline & 2 & (Const.) & 33,18 & 1,61 & & & & \\
\hline & & SS & $-0,27$ & 0,06 & $-0,32$ & 0,15 & $15,57(2,16)$ & 0,001 \\
\hline & & $\mathrm{EC}$ & $-1,25$ & 0,44 & $-0,2$ & & & \\
\hline & 3 & (Const.) & 32,09 & 1,65 & & & & \\
\hline & & SS & $-0,24$ & 0,06 & $-0,29$ & 0,18 & $12,72(3,16)$ & 0,001 \\
\hline & & $\mathrm{EC}$ & $-1,6$ & 0,46 & $-0,25$ & & & \\
\hline & & AS & 0,14 & 0,05 & 0,18 & & & \\
\hline \multirow[t]{2}{*}{ Despersonalización } & 1 & (Const.) & 8,3 & 0,92 & 0,26 & 0,07 & $12,78(1,16)$ & 0,001 \\
\hline & & SAF & 0,13 & 0,03 & & & & \\
\hline \multirow[t]{9}{*}{ Realización Personal } & 1 & (Const.) & 27,32 & 0,57 & 0,18 & 0,03 & $5,86(1,16)$ & 0,017 \\
\hline & & AS & 0,12 & 0,05 & & & & \\
\hline & 2 & (Const.) & 23,47 & 1,81 & 0,18 & 0,06 & $5,48(2,16)$ & 0,005 \\
\hline & & AS & 0,12 & 0,05 & 0,16 & & & \\
\hline & & SPR & 0,15 & 0,06 & & & & \\
\hline & 3 & (Const.) & 24,52 & 1,81 & 0,16 & 0,1 & $6,37(3,16)$ & 0,001 \\
\hline & & AS & 0,1 & 0,04 & 0,31 & & & \\
\hline & & SPR & 0,28 & 0,08 & $-0,25$ & & & \\
\hline & & SAF & $-0,17$ & 0,06 & & & & \\
\hline
\end{tabular}

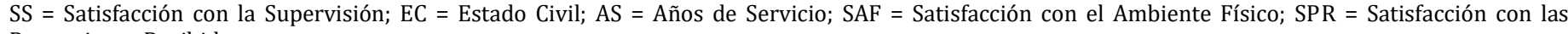
Prestaciones Recibidas 
solteros, estarían más proclives a presentar cansancio emocional.

Por último, para evaluar la consistencia interna de los instrumentos aplicados, se efectuó un análisis de fiabilidad de cada uno de los cuestionarios y sus respectivas dimensiones, apreciándose que las escalas totales del CVP-35 y el S20/23 presentan valores entre 0,8 y 0,9 , lo que significa estar en un rango de bueno/excelente según el criterio planteado por George y Mallery(30). Por su parte, el MBI obtiene un valor cuestionable, aunque siguiendo a Nunnaly(31), resulta ser suficiente cuando sus dimensiones se muestran entre los rangos de 0,66 y 0,72 . Las dimensiones del CVP-35, presentan valores entre 0,39 y 0,83, siendo el más bajo el que corresponde a calidad de vida. Respecto al S20/23, sus dimensiones presentan valores entre 0,82 y 0,93, lo que indica una consistencia interna buena/excelente.

\section{Discusión}

Los datos obtenidos reflejan una prevalencia del SB en los niveles medio/moderado $(88,8 \%)$ y solo un $10 \%$ en los niveles altos, lo cual puede ser entendido desde varias perspectivas(32). En una primera instancia se debe atender al impacto de la sintomatología de mediana intensidad en la respuesta de estrés; que sin manifestarse explícita y agudamente, sugiere su influencia insidiosa $y$ perjudicial en el tiempo. Para el caso del grupo de funcionarios evaluados, ciertas características psicológicas y/o estrategias de afrontamiento que le son tradicionalmente reconocidas por la investigación, como la resiliencia, podrían estar vinculadas al alto porcentaje de SB en los niveles medios, debido a la capacidad preventiva $\mathrm{y} / \mathrm{o}$ amortiguadora de la misma. Las correlaciones más significativas se observan entre las medidas de CVP y SL, implicando una asociación directa entre la vivencia emocional y la valoración cognitiva que los funcionarios hacen de su trabajo, en otras palabras: mientras mayor CVP se manifiesta una mayor SL, sugiriendo así una relación mutua y fortalecedora entre ambas variables. Los resultados obtenidos en este estudio deben ser comprendidos con cierta cautela en el contexto de la aparente contradicción entre los altos niveles de SL y -simultáneamente- una carga laboral excesiva y asociada al moderado y/o alto SB.
Si bien esta relación ha sido abordada por algunos estudios(33), podrían del mismo modo existir otras variables que poseen un factor mediatizador, por ejemplo, la motivación y sentido intrínseco que un trabajador puede darle a su trabajo y contexto laboral.

Disposiciones subjetivas y positivas como un alto compromiso con el rol profesional, apoyan la teoría que estos sujetos se exponen frecuentemente a altas demandas laborales, que usualmente se sobrecargaran en sus esfuerzos y desarrollan una mayor vulnerabilidad a la tensión laboral( ${ }^{34)}$; y solo a largo plazo, a la producción de estrés e insatisfacción(35).

Otra posible respuesta a estos resultados se encuentra en las variables edad y experiencia profesional. Según la literatura en el área, estas se relacionan de un modo directo $\mathrm{y}$ negativo al agotamiento emocional, así como positivo con la eficacia profesional y la implicación laboral(36). La edad cronológica se plantea como un factor predictor del SB, asociado al desarrollo en el tiempo de estrategias de regulación emocional y afrontamiento al estrés, a consecuencia de los años de experiencia en el ejercicio de una profesión determinada, habilidad metacognitiva que mitiga los efectos perjudiciales del SB y la sobrecarga laboral(37). Apoyando parcialmente esta hipótesis, en este trabajo, el rango etario generó diferencias medianamente significativas en la CVP y la Carga de Trabajo, donde los participantes entre 41 y 50 años perciben una mayor carga laboral que aquellos entre los 20 y 30 años; sin embargo, estos últimos perciben más estrés crónico.

Otro resultado destacable se asocian a que los hombres presentan un mayor nivel de Despersonalización y las mujeres más alto en Realización Personal, confirmando la línea de estudio que ha verificado la fuerte vinculación entre las estrategias de afrontamiento, sexo y SL(38). El estado civil genera diferencias en el $\mathrm{SB}$, siendo los funcionarios separados/divorciados quienes presentan los niveles más altos, evidenciando que el disponer de una vinculación emocional de apoyo en la forma de una pareja es un importante factor protector(39). Este hallazgo implicaría tal proclividad por la carencia de personas emocionalmente significativas en la vida del trabajador $\mathrm{y} / \mathrm{o}$ insuficiencia de redes de apoyo efectivas. 
En relación al nivel educacional, se obtuvo diferencias en la dimensión de cansancio emocional, siendo los Técnicos de la salud quienes presentan mayor puntuación. Además, estos presentan una baja percepción de CVP, que según Falgueras, et al.(40), puede asociarse al escaso reconocimiento de su trabajo. Al respecto podría existir una importante variabilidad cultural interesante por investigar en futuros estudios.

Asimismo, se obtuvo relaciones directas entre la realización personal con la carga de trabajo y la motivación intrínseca, siguiendo los hallazgos de Rodríguez, et al.(41), quienes plantean que la realización personal en el trabajo depende de varios factores, algunos intrínsecos como la autopercepción de control de las condiciones laborales proximales, pero también otros de un carácter extrínseco como los recursos en la organización y la carga horaria. Finalmente, se obtienen relaciones indirectas entre Cansancio Emocional y Satisfacción con la Supervisión, similar a los hallazgos de Adriaenssens, et al.(42), los cuales hacen alusión a la influencia del liderazgo de los supervisores en el SB, ya sea como un factor protector o de riesgo, lo cual permite considerar al liderazgo transformacional como un factor protector del SB.

\section{Conclusiones}

Este estudio analizó la asociación entre SB y dos medidas de autopercepción, una emocional asociada al conjunto de sentimientos favorables y desfavorables respecto del trabajo (CVP), y otra cognitiva, asociada a la valoración global sobre la experiencia vital en el trabajo (SL). Estos hallazgos siguen la misma dirección de otros estudios, en relación a la prevalencia específica del SB en la población estudiada, además de la identificación de las variables que pueden ser consideradas de resiliencia para su afrontamiento.

La identificación de estas variables en un factor que ampliamente puede contribuir al diseño de políticas de fomento activo de los recursos laborales y personales de los trabajadores, que en última instancia, deriva en la promoción de su salud mental y crecimiento profesional. De este modo, los resultados obtenidos esperan constituirse en un aporte a la evidencia científica en términos de algunas de las variables de orden individual que poseen un carácter preventivo para la disminución del síndrome de SB, como en este caso específicamente es la edad cronológica y la experiencia laboral.

Variables de corte tradicional en la investigación psicológica como las precisadas en este estudio, invitan a su reconsideración para más específicos trabajos en el área, debido a su poder predictor de las dificultades laborales como es el SB.

La proclividad observada al desarrollo del SB en aquellos sujetos que no poseen relaciones emocionalmente significativas y/o redes de apoyo efectivas, es un tópico relevante de exploración, que se considera debe ser revisado en una mayor profundidad, en vías del apoyo psicosocial que la comunidad laboral puede ofrecer al funcionario con tal situación personal.

Se espera los datos proporcionados contribuyan a la generación de estrategias efectivas de intervención que equilibren las dimensiones cognitivas $y$ emocionales vinculadas a la satisfacción del funcionario de la salud con su entorno laboral, y, en consecuencia, disminuyan los efectos perjudiciales del SB en estas profesiones de alto riesgo. Estrategias organizacionales orientadas a la explicitación simultánea de ambas dimensiones, tanto para el grupo de funcionarios, como para las direcciones, facilitarían la delimitación y trabajo preventivo en los aspectos más objetivos de entorno laboral que contribuyen al desarrollo del SB, de aquellos aspectos matizados por la vida subjetiva del funcionario.

\section{Limitaciones del estudio}

Un diseño longitudinal permitiría conocer no solo el transcurso y los cambios del SB en el tiempo, sino también precisar otras variables que pudiesen tener incidencia en su aparición y/o mantención; debilidad observada con frecuencia en esta línea de estudio. La investigación en esta temática debe responder a las críticas respecto de los criterios de validez y confiabilidad, incorporando evaluaciones mixtas y estadísticas más complejas.

Se sugiere la incorporación de otras variables relevantes, actualmente en exploración empírica, relacionadas a la justicia, compromiso y/o comportamiento de ciudadanía organizacional por la asociación sugerida con la sintomatología ansiosa y satisfacción con el trabajo. 
Asimismo, variables con un carácter preventivo universal sobre el SB podrían ofrecer nuevas luces respecto del síndrome y su afrontamiento efectivo, por ejemplo, la presencia de emociones de carácter compasivo y/o el impacto de las estrategias comunicacionales con los usuarios.

La evidencia sugiere que la organización juega un papel importante en la determinación de los riesgos de salud asociados al SB, por lo que es de interés incorporar variables de la organización específicas a la salud pública de naturaleza comunal, regional y/o nacional. Finalmente, si bien la complejidad del contexto socioeconómico impide por su naturaleza la instauración de cambios expansivos en los entornos laborales, creemos que una visión optimista y basada en la evidencia científica puede significar en el tiempo un aporte concreto a la aproximación efectiva a esta problemática.

\section{Conflicto de interés: Ninguno.}

\section{Referencias}

1. Aronsson G, Theorell T, Grape T, Hammarström A, Hogstedt C, Marteinsdottir I, et al. A systematic review including meta-analysis of work environment and burnout symptoms. BMC Public Health. 2017;17(1):1-13. Disponible en: 10.1186/s12889-017-4153-7

2. Maslach C, Maslach SJ. Lawyer burn-out. Barrister. 1978;5(2):52-54.

3. Díaz-Bambula F, Gómez IC. La investigación sobre el síndrome de Burnout en Latinoamérica entre 2000 y 2011. Rev Psicología del Caribe [revista en Internet] 2016; 33(1): 113-131. Disponible en: http://dx.doi.org/10.14482/psdc.33.1.8065

4. O'Connor K, Muller Neff D, Pitman S. Burnout in mental health professionals: A systematic review and meta-analysis of prevalence and determinants. Eur Psychiatry [Internet]. 2018;53:74-99. Disponible en: https://doi.org/10.1016/j.eurpsy.2018.06.003

5. Rotenstein LS, Torre M, Ramos MA, Rosales RC, Guille C, Sen $\mathrm{S}$, et al. Prevalence of burnout among physicians a systematic review. JAMA - J Am Med Assoc. 2018;320(11):1131-50. Disponible en: https://doi.org/10.1001/jama.2018.12777

6. Bridgeman PJ, Bridgeman MB, Barone J. Burnout syndrome among healthcare professionals. Am J Heal Pharm. 2018;75(3):147-52. Disponible en: https://doi.org/10.2146/ajhp170460

7. Nogueira LS, Sousa RMC, Guedes ES, Santos MAD, Turrini RNT, Cruz DALMD. Burnout and nursing work environment in public health institutions. Rev Bras Enferm. 2018;71(2):336-42. Disponible en: https://doi.org/10.1590/0034-7167-2016-0524

8. Aldrete-Rodríguez MG, Navarro-Meza C, González-Baltazar R, Contreras-Estrada MI, Pérez-Aldrete J. Factores psicosociales y síndrome de burnout en personal de enfermería de una unidad de tercer nivel de atención a la salud. Cienc Trab [Internet]. 2015;17(52):32-6. Disponible en: https://scielo.conicyt.cl/pdf/cyt/v17n52/art07.pdf

9. Párraga-Martínez I, González-Hidalgo E, Méndez-García T, Villarín-Castro A, León-Martín AA. Burnout y su relación con el estrés percibido y la satisfacción laboral en profesionales sanitarios de Atención Primaria de una Comunidad Autónoma. Rev Clínica Med Fam [Internet]. 2018;11(2):51$60 . \quad$ Disponible en: http://scielo.isciii.es/pdf/albacete/v11n2/1699-695Xalbacete-11-02-00051.pdf

10. Maslach C, Schaufeli WB, Leiter MP. Job Burnout. Annual Review of Psychology. 2001;52:397-422. Disponible en: http://dx.doi.org/10.1146/annurev.psych.52.1.397

11. Wilkinson $\mathrm{H}$, Whittington R, Perry L, Eames C. Examining the relationship between burnout and empathy in healthcare professionals: A systematic review. Burn Res [Internet]. 2017;6:18-29. Disponible en: http://dx.doi.org/10.1016/j.burn.2017.06.003

12. Chiang-Vega MM, San Martín-Neira NJ. Análisis de la Satisfacción y el Desempeño Laboral en los Funcionarios de la Municipalidad de Talcahuano. Cienc Trab. 2015;17(54):159-65. Disponible en: http://dx.doi.org/10.4067/S0718-24492015000300001

13. Diener E, Emmons RA, Larsen R, Griffin S. The Satisfaction With Life Scale. Journal of Personality Assessment. 1985;49(1):71-5. Disponible en: http://dx.doi.org/10.1207/s15327752jpa4901_13

14. Veliz Burgos AL, Dörner Paris AP, Soto Salcedo AG, Arriagada Arriagada A. Bienestar psicológico y burnout en profesionales de atención primaria de salud en la región de Los Lagos, Chile. Acta univ [revista en la Internet]. 2018; 28(3):56-64. Disponible en: Disponible en: https://dx.doi.org/10.15174/au.2018.1895

15. Seguel Palma F, Valenzuela Suazo S. Síndrome de burnout en trabajadores de enfermería de dos hospitales del sur de Chile. Av.Enferm. [Internet]. 2016; 34(1): 39-47. http://dx.doi.org/10.15446/av.enferm.v34n1.41599

16. Diario El Centro. El 30\% de los funcionarios del Cesfam la Florida ha tenido licencia médica de tipo siquiátrica [Internet]. Chile: Diario El Centro; 2016. Disponible en: http://www.diarioelcentro.cl/noticias/cronica/el-30-delos-funcionarios-del-cesfam-la-florida-ha-tenido-licenciamedica-de-tipo-siquiatrica?platform=hootsuite

17. Araya VC, Oliva BP, Ananías N, De los Santos P, Mendoza ME. Trastornos Ansiosos y Desórdenes Temporomandibulares en Funcionarios de un Centro de Salud Familiar en la Comuna de Concepción, Chile. Int J Odontostomatol. 2011;5(3):235-9. Disponible en: http://dx.doi.org/10.4067/S0718-381X2011000300005

18. Palma FS, Suazo SV. Síndrome de burnout en trabajadores de enfermería de dos hospitales del sur de Chile. Av en Enfermería [Internet]. 2016;34(1):39-47. Disponible en: https://doi.org/10.15446/av.enferm.v34n1.41599

19. Carmona J, Troncoso P. Satisfacción laboral en funcionarios de salud primaria del a comuna de San Ramón. Santiago: Universidad Academia de Humanismo Cristiano; 2006. Disponible en: http://bibliotecadigital.academia.cl/bitstream/handle/123 456789/1529/ttraso187.pdf?sequence=1\&isAllowed=y

20. Medrano LA, Muñoz-Navarro R, Cano-Vindel A. Procesos cognitivos y regulación emocional: Aportes desde una aproximación psicoevolucionista. Ansiedad y Estres 
[Internet]. 2016;22(2-3):47-54. Disponible en: http://dx.doi.org/10.1016/j.anyes.2016.11.001

21. Sliter MT, McGrew JH, Dreison KC, Salyers MP, Luther L, Bonfils KA. Job burnout in mental health providers: A metaanalysis of 35 years of intervention research. J Occup Health Psychol. 2016;23(1):18-30. Disponible en: http://dx.doi.org/10.1037/ocp0000047

22. Gómez-Perdomo GE, Meneses-Higuita AC, Palacio-Montes MC. Job satisfaction and psychological capital: Factors that affect burnout syndrome. Ansiedad y Estres [Internet]. 2017;23(2-3):71-5. Disponible

en: http://dx.doi.org/10.1016/j.anyes.2017.09.002

23. Mejia CR, Valladares-Garrido MJ, Talledo-Ulfe L, SánchezArteaga K, Rojas C, Arimuya JJ, et al. Síndrome de burnout y factores asociados en estudiantes de medicina: Estudio multicéntrico en siete facultades de medicina peruanas. Rev. chil. neuro-psiquiatr. 2016;54(3): 207-214. Disponible en: http://dx.doi.org/10.4067/S0717-92272016000300005

24. Ferrando J, Pérez J. Un instrumento para medir quemazón profesional en los docentes: adaptación catalana del «Maslach Burnout Inventory (MBI)». Rev Psiquiatr la Fac Med Barcelona. 1996; 23:11-18.

25. Manzanares-Medina E, Merino-Soto C, Fernández-Arata M. Estructura interna del Maslach Burnout Inventory (MBI) en una muestra de sacerdotes y religiosas católicas peruanos. Salud Soc. 2016;7(2):198-211. Disponible en: http://dx.doi.org/10.22199/S07187475.2016.0002.00005

26. Karasek R. PQL-35 questionnaire. Int J Heal Serv. 1989;19(3):481-508.

27. Meliá J, Peiró J. La medida de la satisfacción laboral en contextos organizacionales: El Cuestionario de Satisfacción S20/23. Psicologemas. 1989;5:59-74. Disponible en: https://www.uv.es/ meliajl/Research/Art_Satisf/ArtS20_2 3.PDF

28. Luengo-Martínez C, Lara-Jaque R, López-Espinoza MÁ. Análisis de los componentes principales de la escala Satisfacción laboral (S20-23) medida en una muestra del equipo de enfermería de Centros de Atención Primaria de las Provincias de Ñuble y Valparaíso, Chile. Enfermería global. 2016;15(1):195-207. Disponible en: http://dx.doi.org/10.6018/eglobal.15.1.212481

29. Cohen J. Statistical power analysis for the behavioral sciences. 2nd ed. Hillsdale, NJ: Lawrence Earlbaum Associates; 1988.

30. George D, Mallery P. SPSS for Windows step by step: A Simple Guide and Reference. 11.0 Updat. Boston: Allyn \& Bacon; 2003. Disponible en: https://wps.ablongman.com/wps/media/objects/385/394 732/george4answers.pdf

31. Nunnally NCP. Psychometric Theory. New York: McGrawHill; 1978.
32. de la Fuente EI, García J, Cañadas GA, San Luis C, Cañadas GR, Aguayo R, et al. Psychometric properties and scales of the Granada Burnout Questionnaire applied to nurses. Int J Clin Heal Psychol. 2015;15(2):130-8. Disponible en: https://doi.org/10.1016/j.ijchp.2015.01.001

33. Hilgert JB, Bidinotto AB, Pachado MP, Fara LS, von Diemen L, de Boni RB, et al. Satisfaction and burden of mental health personnel: Data from healthcare services for substance users and their families. Rev Bras Psiquiatr. 2018;40(4):403-9. Disponible en: https://doi.org/10.1590/1516-4446-2017-2352

34. Siegrist J. Adverse Health Effects of High-Effort / LowReward Conditions. J Occup Health Psychol. 1996;1(1):27$41 . \quad$ Disponible en https://pdfs.semanticscholar.org/79da/fe09b439dff93d0cf 4f4a0d231fff321e0a4.pdf

35. Chirico F. Job stress models for predicting burnout syndrome: A review. Ann Ist Super Sanita. 2016;52(3):44356. Disponible en: https://doi.org/10.4415/ANN_16_03_17

36. Johnson SJ, Machowski S, Holdsworth L, Kern M, Zapf D. Edad, estrategias de regulación emocional, burnout e implicación laboral en el sector servicios: ventajas en los trabajadores mayores. Rev Psicol del Trab y las Organ [Internet]. 2017;33(3):205-16. Disponible en: http://dx.doi.org/10.1016/j.rpto.2017.09.001

37. Blanco-Donoso LM, Garrosa E, Demerouti E, MorenoJiménez B. Job resources and recovery experiences to face difficulties in emotion regulation at work: A diary study among nurses. Int J Stress Manag. 2017;24(2):107-34. Disponible en: http://dx.doi.org/10.1037/str0000023

38. Cardoso-da Costa BR, Freire-Pinto ICJ. Stress, Burnout and Coping in Health Professionals: A Literature Review. Psychol Brain Stud [Internet]. 2017;1(1):1-8. Disponible en: https://www.imedpub.com/articles/stress-burnout-andcoping-in-healthprofessionals-a-literature-review.pdf

39. Piñeiro M. Estrés y factores relacionados en el personal sanitario de hospitalización psiquiátrica: un estudio de prevalencia. Enfermería Glob [Internet]. 2013;31:125-50. Disponible en: http://scielo.isciii.es/scielo.php?script=sci_arttext\&pid=S16 95-61412013000300008\&lng=es

40. Falgueras MV, Muñoz CC, Pernas FO, Sureda JC, López MPG, Miralles JD. Burnout y trabajo en equipo en los profesionales de atención primaria. Aten Primaria. 2015;47(1):25-31. Disponible https://doi.org/10.1016/j.aprim.2014.01.008

41. Rodríguez AM, Cruz Pedreros M, Merino JM. Burnout En Profesionales De Enfermería Que Trabajan En Centros Asistenciales De La Octava Región, Chile. Cienc y enfermería. 2009;14(2):75-85. Disponible en: https://scielo.conicyt.cl/pdf/cienf/v14n2/art10.pdf

42. Adriaenssens J, De Gucht V, Maes S. Causes and consequences of occupational stress in emergency nurses, a longitudinal study. J Nurs Manag. 2015;23(3):346-58. Disponible en: https://doi.org/10.1111/jonm.12138 